\title{
Partial Face Recognition
}

\author{
Anuj Rai, Vishnu Agarwal, S. Ushasukhanya
}

\begin{abstract}
Most current Face Identification methods get features and nodes points from still and clear facial images. But, individuals in real world may be obstructed from objects or other people who do not provide the entire image of the person to describe. Keyword-following pats key recognition methods such as: Multiple Key Points with Gbor Triangle (MKD-GTP) and Group Point Matching (RPSM) correspond to the local key points for part of face recognition.Also, on the same note they measure similarity of nodes without higher order mathematical and graphicalinformation that are prone to noise. To solve this, the TPGM method evaluates a static change that encodes the geometry of the other line of the graph, so that a extra precise and stable match with the topology can be calculated.In case to apply higher topological information having higher order, paper offer a topological method to preserve the TPSM algorithm ofconstructionofa higher order shape for each surface and evaluate the change. The paper also suggests that the selection of Viola Jones face recognition points. Deep training can be used to create and combine graphs. This article offers an in-depth study of the progress made in scientific articles and explains the accuracy of the results.
\end{abstract}

\section{INTRODUCTION}

Recognition of the person is a huge area that is currently being investigated by many researchers and suppliers, but there are also some areas that even the researchers have not affected. Partial facial recognition can be seen as one of these areas. Several years ago, researchers thought that partial facial recognition was unreal because there was less information about identifying a person.

According to research by researchers such as Sato et al. (1998), however, indicates that partial facial areas also contain information that can be used for detection. With this project, I have tried to investigate to what extent people need to be recognized in subareas. The artifact allows users to import images of the probe, after which the system determines the face of the person to whom the faces belong. Although it seems like this is a simple process, the system has to do a lot of work.

Investigation of the unique characteristics of the face of the eye, nose and mouth for the detection of individuals. By dividing facial regions, there are fewer unique features that can be used to identify individuals. Identification of unique characteristics of people was archived during the project.Improve Face Recognition Face Recognition features. It is necessary to find the effective algorithm to extract the functions of the segmentation of faces. Apply a robust and efficient facial recognition system based on the facts found in the study. An in-depth study of face detection

Revised Version Manuscript Received on August 19, 2019.

Anuj Rai, B-Tech Scholar, Department of Computer Science and Engineering, SRM Institute of science and Technology, Kattankulathur, India.

Vishnu Agarwal, B-Tech Scholar, Department of Computer Science and Engineering, SRM Institute of science and Technology, Kattankulathur, India.

S. Ushasukhanya, Assistant Professor, Department of Computer Science and Engineering, SRM Institute of science and Technology, Kattankulathur, India. (E-mail: ushasukhanya.s@ktr.srmuniv.ac.in) techniques and algorithms available for partial facial recognition, a selected assessment method and an onboard facial recognition system are being conducted.

\section{LITERATURE SURVEY\& RESULTS}

[1]Topology Preserving Structural Matching for Automatic Partial Face Recognition using TPGM and TPSM:

The first step is to get key points and their description of images of face to create the chart and then to refine the critical important points by rough match. Changing proportion and brightness, which major role in description of key points, the target function for partial adaptation of the face is as follows:

$\min \mathrm{J}=\mathrm{Kt}(\mathrm{t} \mathrm{P}, \mathrm{ht}(\mathrm{t} \mathrm{P}))+\lambda \mathrm{pKp}(\mathrm{f} \mathrm{p}(\mathrm{p} \mathrm{P}), \mathrm{hp}(\mathrm{p} \mathrm{P}))+$ $\lambda q \operatorname{Kq}($ f q (q P ), hq (q P )),

When you add texture matching costs, node matching costs, and edge matching costs, the above formula is calculated for matched TPGM and matched TPSM match Corresponding of topology top order.Comparison of the mean accuracy of the test and the standard deviations (\%) of the different:-

LFW $-74.33 \pm 1.02$

PubFig- $82.98 \pm 1.27$

Drawbacks: The TPGM and TPSM technique was developed to look for and combine the detection and mixing of higher order facial features with partial facial recognition. Because Deep Learning which is the only method to retrieve key-point label, it is very fascinating to use the graphical matching method to increase matching and improve the result. Instead of looking for RPSM, Viola Jones can be used for faster and better results.

[2]Partial Face Recognition: An Alignment Free Approach:

A description of the dimensions of the variables that represent each person with a set of key descriptors. Holistic or partial, the test person's image may be misrepresented by a comprehensive dictionary with writers in the gallery. The procedure is not aligned and addresses key face detection issues through a rapid filtering strategy. To enable partial face recognition, you need to create a large MKD, recognize multiple key SIFT points in each image, and calculate the appropriate descriptors. The MKD-SRC algorithm profitably applied the database of gallery class information and suppressed fraudulent similarity, seeking the most economical representation of all images in the gallery. Then, for all $\mathrm{Yi}$, we separate only the descriptors $\mathrm{L}(\mathrm{L}$ "K) for the larger $\mathrm{L}$ greater number in $\mathrm{Ci}$, which gives a small subdivision D1MXL, then D is replaced by D (i). 
The degree of recognition MKD-SRC in classification 1 is $81.31 \%$ and in SIFT of $58.70 \%$.Drawbacks: The algorithm was unable to detect the faces with very good with illumination problems.Could not detect faces in blur images and images of low quality.

[3] Partial Face Recognition Based on Template Matching:

Recommended approach: Instead of comparing small captions with gallery of front facial images, the locus of point matching algorithm detects features in the gallery within the database which will be the result of most similar image. NCC or cross-correlation which is one of the most popular algorithms based on a correlation, finding the best coincidence coordinates for the maximum value of the mutual correlation among database and test image. This technique is very simple and generates result very fast. The normalization in the composition of NCC causes hindrance to single line variation of illumination. ZNCC and NCC are two similar methods but ZNCC subtracts the mean and is very accurate to match the face model. Local phase quantization is a descriptor of a textured image that is invariant for blur and illumination. Vivante Discrete Transformation which is one of the most robust technique to find image in both frequency and time domain at the same time, finding image sub parts from the image.ar, LFW and FERET collection to match the implementation of the evaluation approach proposal.

On the basis of experimental results, the partial image of the eye results in better recognition accuracy .If you reduce the image size to smaller than $6.25 \%$ of the given full image size, the recognition percentage will be drastically reduced.

Drawbacks: The main drawback is that the algorithm works only on images with good illumination and suitable pixel images. Algorithm may not work with image having conflict area between nose and eyes or having object over face images.

[4] Robust Partial Face Recognition Using Instances-Toclass Distance:

A solid approach to partial face recognition based on the presentation of local resources, where the similarity between each test field and the gallery page surface is calculated using the distance between them and the narrow limitations. SIFT descriptors were derived from partof or still individual images. It can be analysed that the SIFT functions can generatescommon features between the full face and the part of the face. The distance from the distance (I2C) calculates and summarizes distances of all key points from probe image $\mathrm{p}$ and the collection of database images in gallery Gc. After calculating the I2C distance for each and every neighbour from the gallery classifier can be applied to identify.

Accuracy calculation in percentage between LBP and AR having obstruction by handkerchief and glasses.

$\begin{array}{llc}\text { Method } & \text { SIFT } & \text { LBP } \\ \text { I2C (L1) } & 96.17 & 16.50 \\ \text { I2C (L2) } & 91.00 & 12.00\end{array}$

Drawbacks: The method is good to recognize to face but the algorithm does not tell about how it is going to solve the illumination and contrast problem.

[5]Robust Point Set Matching for Partial Face Recognition:
A reliable RPSM method to discriminate these two local collections extracted using attributes local from graphical and text information. The affinity of common parts two faces is changed as the distance between these two ordered sets of functions. For each face image, first identify the key points with a SIFT function detector. Each key point consists of a geometric feature that records its position in the plane of the image and a function that is the descriptor of its characteristic. Here we apply the Lowe mapping scheme to select key points. H. Compare the relationship between the nearest neighbour of the nearest neighbour and the predetermined threshold of a neighbour. These approximate pairs of pairs of pairs are selected for the RPSM for best matching. To align a part of the probe with a gallery, the corresponding geometrical and structural features must be adjusted simultaneously. The matching process has the following three characteristics:

- Adjust the sub-setting.

- personal correspondence.

- Non-Aphonic Transformation.

Verification accuracy on LFW dataset:-

RPSM-SiftSurfSILBP $0.7165 \pm 0.0157$

Verification accuracy on Pubfig dataset:-

RPSM-SiftSurfSILBP $0.7889 \pm 0.0175$

Drawbacks: The distinctive feature of RPSM is its robust matching scheme, which examines both geometric distribution consistency and textural similarity.

[6] A study on partial face recognition of eye region:

This article uses a partial space positioning to reduce the capacity of given image, keeping as many strokes as possible. Facial images are run across non-negative matrix (MFN), local NMF (LNMF) and spatially confined MFN (SFNMF) factorization.The NMF finds approximate part of value, where $\mathrm{X}$ represents the raw data for the person with values of $\mathrm{W}$ and $\mathrm{H}$ which are nonnegative in nature. The upper bound of minus make the performance purely summarizing (without subtraction), unlike other algorithm like PCA which uses single continues representation. This confirms that different parts are unified in a non-subtractive way. The LNMF is applied to increase the localization by applying restrictions which is learned by resources. The SFNMF base image represents only spatially restricted regions. This is a very creative idea of part identificationin the place where the spatially constrained regions 48 correspond.

It is observed that the LNMF reaches an optimal detection, which is $95.12 \%$, followed by SFNMF and MFN, reaching $94.48 \%$ and $93.23 \%$, respectively. It should be noted that there is a certain decrease of $1.2 \%, 1.89 \%$ and $2.24 \%$ in relation to the complete person for MFN, LNMF and SFNMF.

Drawbacks: Blinking of eye increases error rate.This paper only deals with eye region and not with other features of faces which may be even more challenging.

[7] Face Recognition with Patterns of Oriented Edge Magnitudes: 
This article addresses the problem of low-cost, yet differentiating and robust face recognition features in real world. The introduced descriptor has the properties which are required: POEM (1) is a multidimensional spatial descriptor that captures comprehensive information about the original image. (2) is a multidimensional structure based on self-interest and leading to resistance to external fluctuations; and (3) is of low complexity and therefore very easy to apply for real-time applications. In summary, for each pixel, the POEM function is constructed by applying a self-imposed structure based on a dimension calculated by the accumulation of a local gradient orientation histogram over all pixels of the images centered on the pixel in question. The persistence and discrimination power of the POEM descriptor is evaluated for the facial recognition of bounded (FERET) and unrestricted (LFW) data sets. Experimental results show that this algorithm works better than most other algorithms. Most impressively, the cost of calculating to retrieve the POEM descriptor is so low that it runs about 20 times faster than the first step of the Gabor filter methods. In addition, memory which is needed is 13 and 27 times less than that of LGBP (Local Gabor Binary Patterns) and HGPP (Gabor Phase Chart Histogram) standards.Drawbacks: Future work will include testing the POEM descriptor on a different problems of computer vision tasks, such as Computer Vision, Face Recognition and Object Recognition. This can also be used to investigate the use of more powerful classifiers than the POEM descriptor in the facial recognition domain.

[8]Face Description with Local Binary Patterns:

Face Detection Program: This document presents a new and effective representation of the person's image on the basis of texture characteristics of the local binary model (LBP). The facial image is subdivided into several parts, from which the LBP key points are taken and linked into an extended feature vector that will be applied as face descriptor. The implementation of this method is evaluated as a problem of identifying individuals in various challenges. Different implementation and changes which are needed to added are also discussed.

Drawbacks: This work may include the study of much more realistic method of subdividing and dividing images into local areas and finding the weight for those parts. The AdaBoost can be taken as a good research base. Another good field is the search for methods and descriptors for image preprocessingwhich are not prone to resistance change and can change the texture of surface.

[9]On the use of SIFT features for face authentication:

Different techniques for recognizing and classifying images are used in biometrics. An interesting technique is the large-scale invariant transformation (SIFT), which was initially designed for object recognition. Although SIFT functions appear to be very powerful image descriptors, their use in the context of facial analysis has never been systematically investigated.

This paper examines the implementation of the SIFT approach in the context of the person's authenticity. To determine the true potential and applicability of the method, different matching schemes with BANCA and the protocol are offered and tested to show promising results.
Drawbacks:When you use the SIFT functions for the first time, it appears that the matching feature process must be controlled to match the face's variability and format features. On the other hand, the SIFT algorithm itself needs to be analyzed and adjusted to be fully adapted to the shape and structure of the person.

[10] Regularized Robust Coding for Face Recognition:

Recently, a rare classification (SRC) for strong facial recognition (RF) was proposed. In SRC, the given image is translated into rare continues mixture of training patterns, and performance correctness is calculated with either a 12node or a coding residue standard. In such a diluted coding model, it can be said that leftover image follows Gauss and Laplace distribution and may not be acceptable to explain the coding leftover in practical FR systems. At the same time upper bound of coding coefficient uniqueness increases the overall SRC cost. This paper describes the new Facial Coding Model, that is, RRC, which can continuously recede a given signal with balanced regression coefficients. Supposing that the coding and scrambling coefficient is not dependent and similarly parted, the RRC search for at most a next similar solution to the coding problem. An repetitive well adjusted re-balanced stable coding algorithm (I R3C) is introduced to effectively solve the RRC model.The in depth experimentation shows that RRC method is much more capable than the most recent, rare and representative methods of dealing with closing, corruption, lighting and phrase changes, and so on.

Drawbacks: The RRC methods suggested were extensively evaluated in RF with different conditions, including variations in illumination, expression, occlusion, corruption and face validation. But this method requires more computational power and resources.

\section{CONCLUSION}

This literature survey provides with various method to recognise face and alsotheiraccuracy. And as we see from the report above different techniques have tried to solve the puzzle of face identification but most of them come short when it comes to occluded face or partial face or simply when the picture is just not ideal but partial face matching a completely new domain fulfils most of the forthcoming.

\section{REFERENCES}

1. Topology Preserving Structural Matching for Automatic Partial Face Recognition;YueqiDuan, Jiwen Lu, Jianjiang Feng, JieZhouS. R. Arashloo, J. Kittler, "Efficient processing of MRFs for unconstrained-pose face recognition", Proc. BTAS, pp. 1-8, 2013

2. H. Bay, A. Ess, T. Tuytelaars, L. Van Gool, "Speeded-up robust features (SURF)", Comput. Vis. Image Understand., vol. 110, no. 3, pp. 346-359, 2008

3. P. N. Belhumeur, J. P. Hespanha, D. Kriegman, "Eigenfaces vs Fisherfaces: Recognition using class specific linear projection", IEEE Trans. Pattern Anal. Mach. Intell, vol. 19, no. 7, pp. 711-720, Jul. 1997.

4. Partial Face Recognition: An Alignment Free Approach; Shengcai Liao, Anil K. JainG. Hua, M.-H. Yang, E. Learned-Miller, Y. Ma, M Turk, D.J. Kriegman, T.S. Huang, "Introduction to the Special Section on Real-World Face Recognition", IEEE Trans. Pattern Analysis and Machine Intelligence, vol. 33, no. 10, pp. 1921-1924, Oct. 2011. 
5. G.B. Huang, M. Ramesh, T. Berg, E. Learned-Miller, "Labeled Faces in the Wild: A Database for Studying Face Recognition in Unconstrained Environments", Technical Report 07-49, Oct. 2007, [online] Available: http://vis-www.cs.umass.edu/lfw/.

6. Partial Face Recognition Based on Template Matching; SoodehNikan , Majid Ahmadi.T. F. Cootes, C. J. Taylor, D. Cooper, J. Graham, "Active shape models - their training and application", Computer Vision and Image Understanding, vol. 61, pp. 38-59, Jan. 1995.

7. L. Di Stefano, S. Mattoccia, F. Tombari, "Comparison of Various Template Matching Techniques for Face Recognition", International Journal of Engineering Research and Development, vol. 8, pp. 16-18, Oct. 2013.

8. Robust Partial Face Recogintion Using Instances-To-class Distance;JunlinHu,JiwenLu,Yap-PengTanW. Zhao, R. Chellappa, P Phillips, and A. Rosenfeld, "Face recognition: A literature survey", ACM Computing Surveys, vol. 35, no. 4, pp. 399-458, 2003.

9. J. $\mathrm{Lu}$ and Y.-P. Tan, "A doubly weighted approach for appearancebased subspace learning methods", IEEE Transactions on Information Forensics and Security, vol. 5, no. 1, pp. 71-81, 2010

10. Robust Point Set Matching for Partial Face Recognition; RenliangWeng, Jiwen Lu, Yap-Peng TanM. A. Turk, A. P. Pentland, "Face recognition using eigenfaces", Proc. CVPR, pp. 586-591, Jun. 1991.

11. M. S. Bartlett, J. R. Movellan, T. J. Sejnowski, "Face recognition by independent component analysis", IEEE Trans. Neural Netw., vol. 13, no. 6, pp. 1450-1464, Nov. 2002.

12. A study on partial face recognition of eye region; Chuan Chin Teo,Han Foon Neo, Andrew Beng Jin Teoh.H. J. Oh, K. M. Lee, S. U. Lee, "Occlusion invariant face recognition using selective LNMF basis images", Proc. Asian Conf. on Computer Vision (ACCV 2006), Jan. 2006.

13. M. Savvides, R. Abiantun, J. Heo, C. Xie, B.V.K Vijayakumar, "Partial \& Holistic Face Recognition on FRGC-II data using Support Vector Machine", Proc. on Computer Vision and Pattern Recognition Work-shop (CVPRW 2006), pp. 48, 2006.

14. Enhanced Patterns of Oriented Edge Magnitudes for Face Recognition and Image MatchingN.-S. Vu, A. Caplier, "Face recognition with patterns of oriented edge magnitudes", Proc. ECCV, pp. 313-326, 2010.

15. V. Perlibakas, "Distance measures for PCA-based face recognition", Pattern Recognit. Lett., vol. 25, no. 6, pp. 711-724, Apr. 2004.

16. "Face Description with Local Binary Patterns": Application to Face Recognition TimoAhonen, Student Member, IEEE, AbdenourHadid, and MattiPietikainen, Senior Member, IEEEArkoLucieer, Alfred Stein, Peter Fisher, Multivariate Texture-based Segmentation of Remotely Sensed Imagery for Extraction of Objects and Their Uncertainty.

17. K. Etemad, R. Chellappa, "Discriminant Analysis for face recognition of Human Face images", I.Optical Soc. Am., vol. 14, pp. 1724-1733, 1997.

18. "Face Description with Local Binary Patterns": Application to Face Recognition TimoAhonen, Student Member, IEEE, AbdenourHadid, and MattiPietikainen, Senior Member, IEEE:E. Bailly-Baillire, S. Bengio, F. Bimbot, M. Hamouz, J. Kittler, J. Marithoz, J. Matas, K. Messer, V. Popovici, F. Pore, B. Ruiz, and J.-P. Thiran. The BANCA database and evaluation protocol. In Proc. Int. Conf. on Audio- and VideoBased Biometric Person Authentication (AVBPA03), pages 625-638. Springer-Verlag, 2003.

19. M. Bertero, T. Poggio, and V. Torre. Ill-posed problems in early vision. Proceedings of the IEEE, 76(8):869-889, Aug. 1988.

20. Regularized Robust Coding for Face Recognition , Meng Yang, Member, IEEE, Lei Zhang, Member, IEEE, Jian Yang, Member, IEEE, and David Zhang, Fellow, IEEE.

21. B. Heisele, P. Ho, and T. Poggio, "Face recognition with support vector machine: Global versus component-based approach," in Proc. IEEE Int. Conf. Comput. Vis., Aug. 2001, pp. 688-694.

22. S. Chen, T. Shan, and B. C. Lovell, "Robust face recognition in rotated eigenspaces," in Proc. 22nd Int. Conf. Image Vis. Comput., 2007, pp. 1-6. 